Chapter 6

\title{
Unsteady Axial Viscoelastic Pipe Flows of an Oldroyd B Fluid
}

\author{
A. Abu-El Hassan and E. M. El-Maghawry \\ Additional information is available at the end of the chapter \\ http://dx.doi.org/10.5772/53638
}

\section{Introduction}

The unsteady flow of a fluid in cylindrical pipes of uniform circular cross-section has applications in medicine, chemical and petroleum industries [3,4,5]. For viscoelastic fluids, the unsteady axial decay problem for UCM fluid is considered by Rahman et al. [6]; and for Newtonian fluids as a special case. Rajagopal [7] has studied exact solutions for a class of unsteady unidirectional flows of a second-order fluid under four different flow situations. Atalik et al. [8] furnished a strong numerical evidence that non-linear Poiseuille flow is unstable for UCM, Oldroyd-B and Giesekus models. This fact is supported experimentally by Yesilata, [9]. The unsteady flow of a blood, considered as Oldroyd-B fluid, in tubes of rigid walls under specific APGs is concerned by Pontrelli, [10, 11].

Flow of a polymer solution in a circular tube under a pulsatile APG was investigated by Barnes et al. [12, 13].The same problem for a White-Metzner fluid is performed by Davies et al. [14] and Phan-Thien [15]. Recently, periodic APG for a second-order fluid has been studied by Hayat et al. [16]. Numerical simulation based on the role of the pulsatile wall shear stress in blood flow, is investigated by Grigioni et al. [1].

The present paper is concerned with the unsteady flow of a viscoelastic Oldroyd-B fluid along the axis of an infinite tube of circular cross-section. The driving force is assumed to be a time-dependent APG in the following three cases:

i. APG varies exponentially with time,

ii. Pulsating APG,

iii. A starting flow under a constant APG. 


\section{Formulation of the problem}

The momentum and continuity equations for an incompressible and homogenous fluid are given by

$$
\rho \frac{d q}{d t}=-\nabla P+\nabla \cdot \underline{\underline{S}}
$$

and

$$
\nabla \cdot \underline{q}=0
$$

where $\varrho$ is the material density, $q$ is the velocity field, $\mathrm{p}$ is the isotropic pressure and $S$ is the Cauchy or extra-stress tensor. The constitutive equation of Oldroyd-B fluid is written as

$$
\left.\underline{T}=-p \underline{\underline{I}}+S ; \underline{\underline{S}}+\lambda_{1} \underline{\underline{S}}=\mu \underline{\underline{A_{1}}}+\lambda_{2} \underline{\underline{A_{1}}}\right\}
$$

where $\underline{\underline{T}}$ is the total stress, $\underline{\underline{I}}$ is the unit tensor, $\mu$ is a constant viscosity, $\lambda_{1}$ and $\lambda_{2},\left(0 \leq \lambda_{2} \leq \lambda_{1}\right)$ are the material time constants, termed as relaxation and retardation times; respectively. The deformation tensor $\mathrm{A}_{1}$ is defined by

$$
\underline{\underline{\mathrm{A}}}=\underline{\underline{L}}+\underline{\underline{L}} \underline{\underline{T}}_{\underline{L}}^{\underline{L}}=\nabla \underline{q}
$$

and " $\nabla$ " denotes the upper convected derivative ; i.e. for a symmetric tensor $\underline{\underline{G}}$ we get,

$$
\stackrel{\nabla}{=}=\frac{\partial \underline{\underline{G}}}{\partial t}+\underline{q} \cdot \nabla \underline{\underline{G}}-\underline{\underline{G}} \cdot \underline{\underline{L}}-\underline{L^{T}} \cdot \underline{\underline{G}}
$$

The symmetry of the problem implies that $\underline{\underline{S}}$ and $q$ depend only on the radial coordinate $\mathrm{r}$ in the cylindrical polar coordinates $(r, \theta, z)$ where the $z$-axis is chosen to coincide with the axis of the cylinder. Moreover, the velocity field is assumed to have only a $\mathrm{z}$-component, i.e.

$$
\underline{q}=(0,0, \underline{w})
$$

which satisfies the continuity equation (2) identically. The substitution of Eq. (6), into Eqs. (1) and (3) yields the set of equations 


$$
\begin{gathered}
S_{r z}+\lambda_{1} \frac{\partial S_{r z}}{\partial t}=\mu\left(\frac{\partial w}{\partial r}+\lambda_{2} \frac{\partial^{2} w}{\partial r \partial t}\right), \\
\frac{\partial p}{\partial z}=\frac{\partial S_{r z}}{\partial r}+\frac{1}{r} S_{r z}-\rho \frac{\partial w}{\partial t} \\
\frac{\partial p}{\partial r}=\frac{\partial p}{\partial \theta}=0 .
\end{gathered}
$$

Equations (8) and (9) imply that the pressure function takes the form; $p=z f(t)+c$, so that

$$
\frac{\partial p}{\partial z}=f(t)
$$

The elimination of $S_{r z}$ from (7) and (8) shows that velocity field $w(r, t)$ is governed by:

$$
\rho\left(\lambda_{1} \frac{\partial^{2} w}{\partial t^{2}}+\frac{\partial w}{\partial t}\right)-\mu\left(1+\lambda_{2} \frac{\partial}{\partial t}\right)\left(\frac{\partial^{2} w}{\partial t^{2}}+\frac{1}{r} \frac{\partial w}{\partial r}\right)=-\left(1+\lambda_{1} \frac{\partial}{\partial t}\right) \frac{\partial p}{\partial z}
$$

The non-slip condition on the wall and the finiteness of $w$ on the axis give

$$
\left.w(r, t)\right|_{r=R}=0 \text { and }\left.\frac{\partial w}{\partial r}\right|_{r=0}=0
$$

Introducing the dimensionless quantities

$$
\eta=\frac{r}{R}, \tau=\frac{\mu t}{\rho R^{2}}, \varphi=\frac{\mu L}{\Delta P R^{2}} w, \lambda=\frac{\lambda_{2}}{\lambda_{1}} \text { and } H=\frac{\lambda_{1} \mu}{\rho R^{2}}=\frac{W e}{\operatorname{Re}}
$$

where $\mathrm{R}$ is the radius of the pipe, $\Delta P$ a characteristic pressure difference, $\mathrm{L}$ is a characteristic length, We and Re are the Weissenberg and Reynolds numbers; respectively, into Eqs. (10), (11) and (12) we get

$$
H \frac{\partial^{2} \varphi}{\partial \tau^{2}}+\frac{\partial \varphi}{\partial \tau}-\left[1+\lambda H \frac{\partial}{\partial \tau}\right]\left[\frac{\partial^{2} \varphi}{\partial \eta^{2}}+\frac{1}{\eta} \frac{\partial \varphi}{\partial \eta}\right]=\left[1+H \frac{\partial}{\partial \tau}\right] \Psi(\tau)
$$


with the BCs.

$$
\varphi(1, \tau)=0 \text { and } \frac{\partial \varphi(0, \tau)}{\partial \eta}=0
$$

and

$$
\Psi(\tau)=-\frac{L}{\Delta p} \frac{\partial p}{\partial z}=-\frac{L}{\Delta p} f(t) .
$$

Equation (14) subject to BCs. (15) is to be solved for different types of APGs; i.e. different forms of the function $\Psi(\tau)$.

\section{Pressure gradient varying exponentially with time}

We consider the two cases of exponentially increasing and decreasing with time APGs separately.

\subsection{Pressure gradient increasing exponentially with time}

Let,

$$
\Psi(\tau)=-\frac{L}{\Delta p} \frac{\partial p}{\partial z}=K e^{\alpha^{2} \tau}
$$

and assume that

$$
\varphi(\eta, \tau)=g(\eta) e^{\alpha^{2} \tau}
$$

where $K$ and $\alpha$ are constants. The substitution of Eqs. (17) and (18) into Eq. (14) leads to

$$
g^{\prime \prime}+\frac{1}{\eta} g^{\prime}-\frac{\alpha^{2}\left(H \alpha^{2}+1\right)}{\lambda H \alpha^{2}+1} g=-K \frac{H \alpha^{2}+1}{\lambda H \alpha^{2}+1}
$$

while the BCs. (15) reduce to 


$$
g(1)=0, g^{\prime}(0)=0
$$

A solution of Eq. (19) subject to the BCs. (20) is

$$
g(\eta)=\frac{K}{\alpha^{2}}\left[1-\frac{I_{0}(\beta \eta)}{I_{0}(\beta)}\right]
$$

where $\mathrm{I}_{0}(\mathrm{x})$ is the modified Bessel-functions of zero-order, and

$$
\beta^{2}=\frac{\alpha^{2}\left(1+H \alpha^{2}\right)}{1+\lambda H \alpha^{2}} .
$$

Therefore, the velocity field is given by

$$
\varphi(\eta, \tau)=\frac{K}{\alpha^{2}}\left[1-\frac{I_{0}(\beta \eta)}{I_{0}(\beta)}\right] e^{\alpha^{2} \tau} .
$$

The solution given by Eq. (23) processes the following properties:

i. The time dependence is exponentially increasing such that for $\eta \neq 1 \lim _{\tau \rightarrow \infty} \phi(\eta, \tau) \rightarrow \infty$. It may be recommendable to choose another APG which increases up to a certain finite limit in order to keep $\phi(\eta, \tau)$ finite.

ii. The present solution depends on the parameter $\beta$ in the same form as the solution for the UCM [6]. For any value of $\beta$ the Oldroyd-B fluid exhibits the same form as the UCM- fluid. However, in the present case $\beta$ depends on $\lambda$ in addition to $\mathrm{H}$ and $\alpha^{2}$. A close inspection show that $\lim _{\lambda \rightarrow 0} \beta^{2}=\beta^{2}$ for the UCM-fluid while the $\lim _{\lambda \rightarrow 1} \beta^{2}=\alpha^{2}$ which coincides with the case of the Newtonian fluid, [8].

iii. The parameter $\beta$ is inversely proportional to $\lambda$ where the decay rate increases by increasing the value of $\mathrm{H}$. However, as mentioned above, as $\lambda$ approaches the value $\lambda=1$ all the curves matches together approaching the value $\beta^{2}=\alpha^{2}$ asymptotically. The behavior of $\beta$ as a function of $\lambda$, where $H$ is taken as a parameter is shown in Fig. (1).

For small values of $|\beta|$ and by using the asymptotic expansion of $\mathrm{I}_{0}(\mathrm{x})$,

it can be shown that the velocity profiles approaches the parabolic distribution;

$$
\operatorname{Lim}_{\beta \rightarrow 0} \varphi(\eta, \tau)=\frac{K\left(H \alpha^{2}+1\right)}{4\left(\lambda H \alpha^{2}+1\right)}\left(1-\eta^{2}\right) e^{\alpha^{2} \tau}
$$




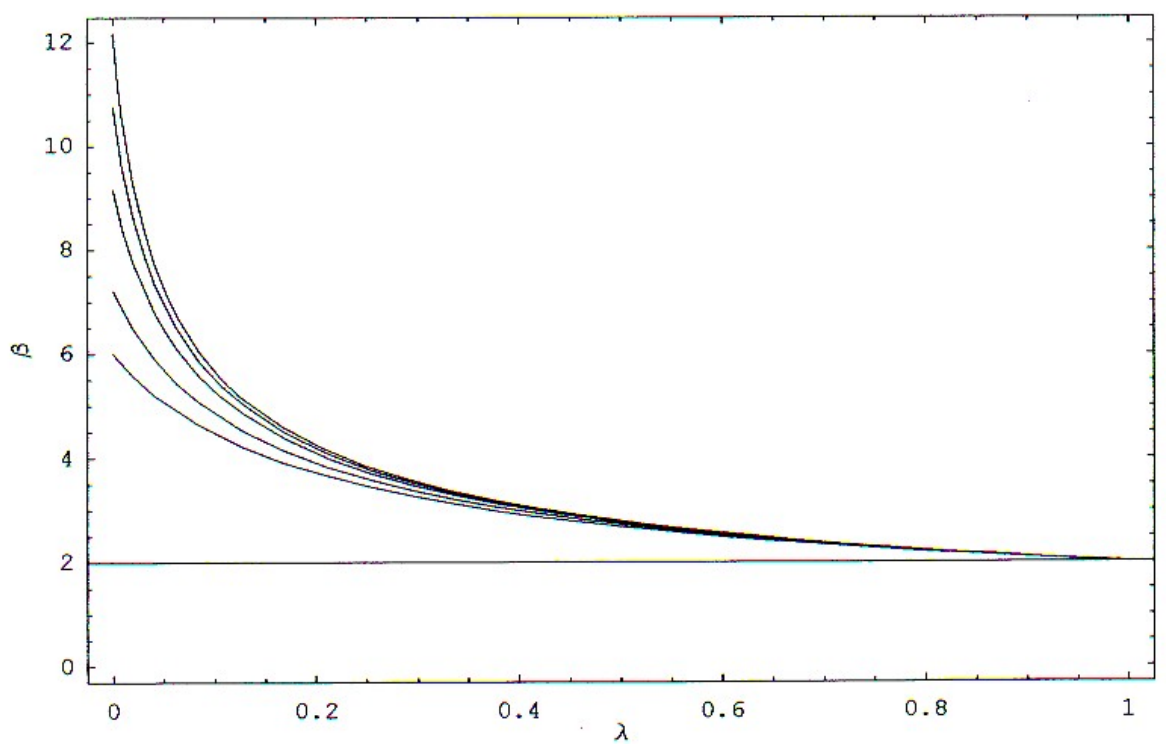

Figure 1. The $\lambda \beta$ - relation $\mathrm{H}=2,3,5,7,9$, (Bottom to top)

For the case of large $|\beta|$ the velocity distribution is given as;

$$
\operatorname{Lim}_{\beta \rightarrow \infty} \varphi(\eta, \tau)=\frac{K}{\alpha^{2}}\left[1-\frac{1}{\sqrt{\eta}} e^{-\beta(1-\eta)}\right] e^{\alpha^{2} \tau}
$$

This solution is completely different from the parabolic distribution and it depends on $\eta$ only in the neighborhood of the wall. Therefore, such a fluid exhibits boundary effects.

The rising-APG velocity field $\phi(\eta, \tau)$ is plotted in Figs. (2a) and (2b) as a function of $\eta$ at different values of $\beta$ for $\alpha=2$ and $\alpha=5$.

\subsection{Pressure gradient decreasing exponentially with time}

The solution at present is obtained from the previous case by changing $\alpha^{2}$ by $-\alpha^{-2}$. Therefore,

$$
\varphi(\eta, \tau)=-\frac{K}{\alpha^{2}}\left[1-\frac{j_{0}\left(\beta_{1} \eta\right)}{j_{0}\left(\beta_{1}\right)}\right] e^{-\alpha^{2} \tau} .
$$

where 


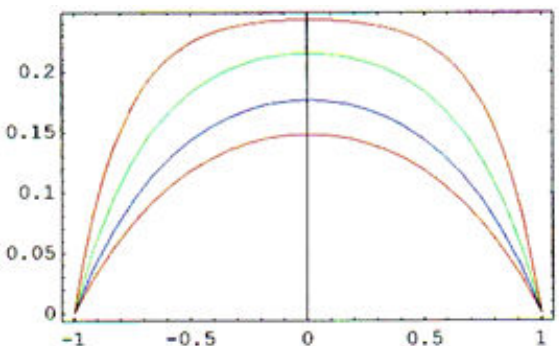

(a)

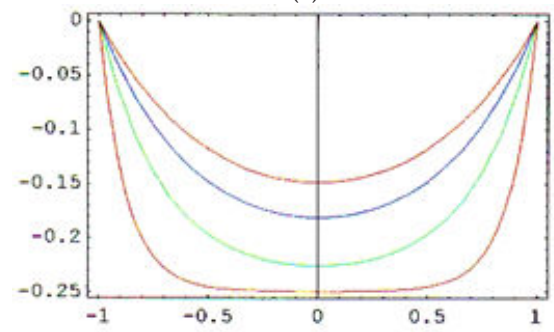

(c)

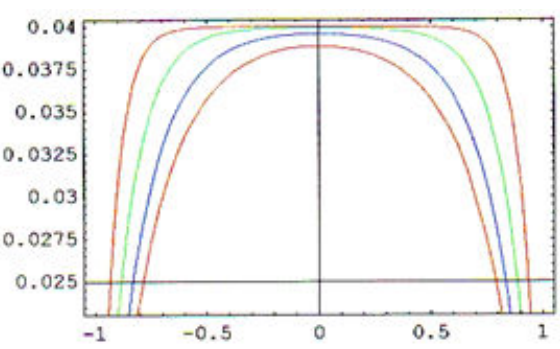

(b)

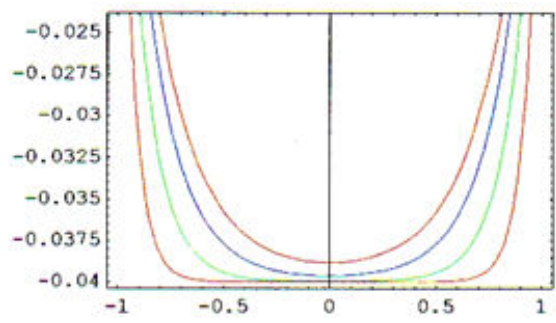

(d)

Figure 2. Rising - $(a, b)$ APG velocity filed $\beta=5.2,3.5,2.5,2.1$ (Bottom to top) Fig. (c) : Decreasing - APG velocity filed $; \beta=8.7,3.9,2.6,2.1$ ( Top to Bottom) Fig. (d) : Decreasing - APG velocity filed ; $\beta=16.4,9.2,6.5$, 5.3 ( Top to Bottom)

$$
\beta_{1}^{2}=\frac{\alpha^{2}\left(1-H \alpha^{2}\right)}{1-\lambda H \alpha^{2}}
$$

The discussion of this solution is similar to the case of increasing APG except that the velocity decays exponentially with time and the value $\alpha^{2}=1 / \lambda H$ is not permissible as it leads to infinite $\beta_{1}^{2}$; i.e.

$$
\lim _{\alpha_{1}^{2} \rightarrow 1 / \lambda H} \beta_{1}^{2} \rightarrow \infty
$$

The two cases of small and large $\left|\beta_{1}\right|$ produce similar results as the previous solution. Thus

$$
\operatorname{Lim}_{\beta_{1} \rightarrow 0} \phi(\eta, \tau)=-\frac{K}{4 \alpha^{2}} \beta_{1}^{2}\left(1-\eta^{2}\right) e^{-\alpha \tau}
$$

and 


$$
\operatorname{Lim}_{\beta_{1} \rightarrow \infty} \varphi(\eta, \tau)=-\frac{K}{\alpha^{2}}\left[1-\frac{1}{\sqrt{\eta}} \frac{\cos \left(\beta_{1} \eta-\frac{\pi}{4}\right)}{\cos \left(\beta_{1}-\frac{\pi}{4}\right)}\right] e^{-\alpha^{2} \tau} .
$$

\section{Pulsating pressure gradient}

The present case requires the solution of Eq. (14) subject to BCs. (15) in the form

$$
\Psi(\tau)=-\frac{L}{\Delta P} \frac{\partial p}{\partial z}=K e^{i n \tau} ; \quad i=\sqrt{-1}
$$

$\mathrm{K}$ and $\mathrm{n}$ are constants. Assuming the velocity function has the form

$$
\begin{gathered}
\varphi(\eta, \tau)=r e\left[f(\eta) e^{i n \tau}\right] \\
\therefore \mathrm{f}^{\prime \prime}+\frac{1}{\eta} \mathrm{f}^{\prime}-i n \frac{(1+i n H)}{(1+i n \lambda H)} \mathrm{f}=-K \frac{(1+i n H)}{(1+i n \lambda H)} .
\end{gathered}
$$

The solution of this equation satisfying the BCs. (15) is :

$$
\mathrm{f}(\eta)=\frac{k}{i n}\left[1-\frac{I_{0}(\beta \eta)}{I_{0}(\beta)}\right], \quad \beta^{2}=i n \frac{(1+i n H)}{(1+i n \lambda H)}
$$

Hence, the velocity distribution is given by:

$$
\phi(\eta, \tau)=\operatorname{re}\left\{\frac{k}{i n} e^{i n \tau}\left[1-\frac{I_{0}(\beta \eta)}{I_{0}(\beta)}\right]\right\} .
$$

Obviously; for small $|\beta|$,

$$
\operatorname{Lim}_{\beta \rightarrow 0} f(\eta)=\frac{K}{i n}\left(\frac{\beta^{2}\left(1-\eta^{2}\right)}{4}\right)
$$

and for large $|\beta|$ 


$$
\operatorname{Lim}_{\beta \rightarrow \infty} \frac{I_{0}(\beta \eta)}{I_{0}(\beta)}=\frac{1}{\sqrt{\eta}} e^{-\beta(1-\eta)},
$$

So that,

$$
\varphi(\eta, \tau)=\operatorname{re}\left\{\frac{K}{i n}\left[1-\frac{1}{\sqrt{\eta}} e^{-\beta(1-\eta)} e^{i n \tau}\right]\right\},
$$

where,

$$
\beta^{2}=\frac{i n(1+i n H)}{(1+i n \lambda H)}=\frac{1}{1+n^{2} \lambda^{2} H^{2}}\left[n^{2} H(\lambda-1\}+i n\left(1+n^{2} \lambda H^{2}\right)\right]
$$

or simply,

$$
\begin{gathered}
\beta=\sqrt{\Re} e^{i \theta / 2}, \\
\Re=\frac{n}{1+n^{2} \lambda^{2} H^{2}} \sqrt{n^{2} H^{2}(1-\lambda)^{2}+\left(1+n^{2} \lambda H^{2}\right)^{2}}, \\
\frac{\theta}{2}=-\frac{1}{2} \operatorname{Tan}^{-1}\left[\frac{1+n^{2} \lambda H}{n H(1-}\right] .
\end{gathered}
$$

Substituting from Eqs.(40,41,42) into Eq. (37), we get:

$$
\operatorname{Lim}_{\beta \rightarrow \infty} \varphi(\eta, \tau)=\frac{k}{n}\left\{\sin n \tau-\frac{1}{\sqrt{\eta}} e^{-(1-\eta) \sqrt{\Re} \cos (\theta / 2)} \sin \left[n \tau-\left(1-\eta \sqrt{\Re} \sin \frac{\theta}{2}\right)\right]\right\} .
$$

As $\lambda \rightarrow 0,[6], \mathfrak{R} \rightarrow r_{1}=n \sqrt{1+n^{2} H^{2}}$ and $\frac{\theta}{2} \rightarrow \frac{\theta_{1}}{2}=-\frac{1}{2} \operatorname{Tan}^{-1}\left(\frac{1}{n H}\right)$.

Then

$$
\varphi(\eta, \tau)=\frac{k}{n}\left\{\sin n \tau-\frac{1}{\sqrt{\eta}} e^{-(1-\eta) \sqrt{r_{1}}\left(\cos \theta_{1} / 2\right)} \sin \left[n \tau-(1-\eta) \sqrt{r_{1}}\left(\sin \frac{\theta_{1}}{2}\right)\right]\right\}
$$

The velocity field $\varphi(\eta, \tau)$ is plotted in Figs. (3a) and (3b); respectively, against $\eta$ for different values of $\beta$. The two limiting cases for small and large $|\beta|$ are represented in three-dimensional Figs. (4a) and (4b) in order to emphasize the oscillating properties of the solution. 


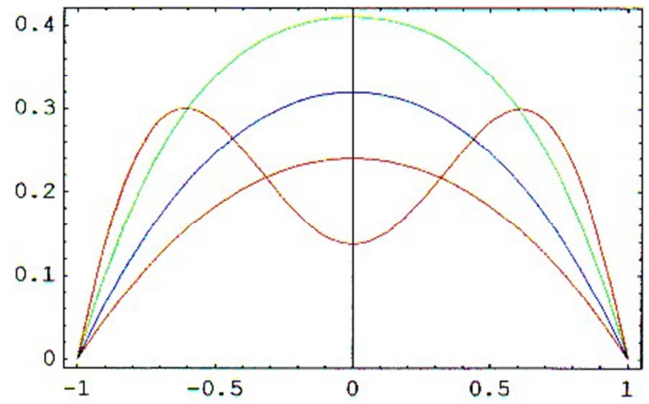

(a)

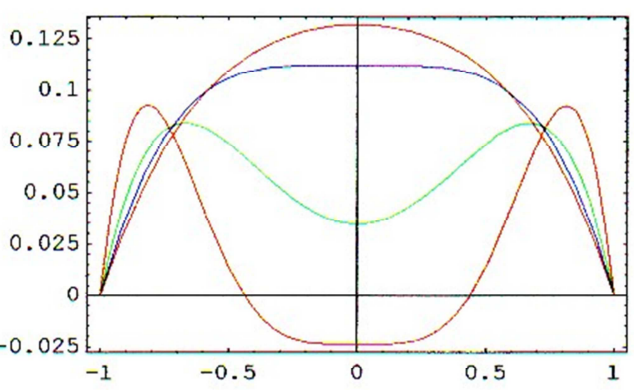

(b)

Figure 3. a) : Pulsating - APG ; $n=2, H=5, \beta=3.7,2.5,1.8,1.5$ (b) : Pulsating - $A P G ; n=5, H=5$, $\beta=6.8,4.1,2.9,2.4$ [Top to Bottom for all]
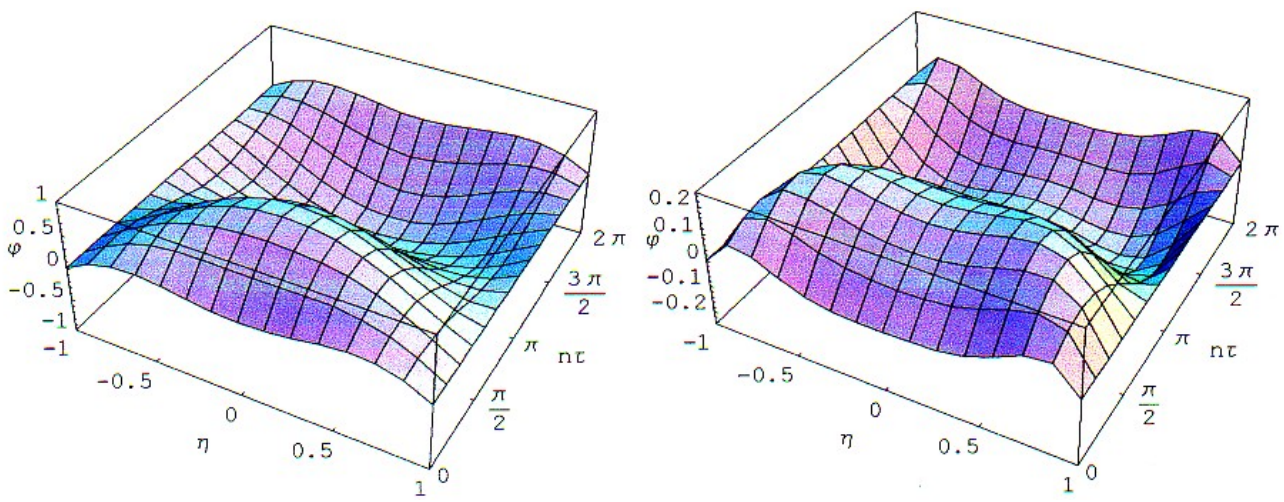

Figure 4. a): Pulsating-APG, $n=2, H=5$, at small $|\beta| ; \beta=3.7$ (b): Pulsating-APG, $n=3, H=5$, at large $|\beta| ; \beta=6.8$

\section{Constant pressure gradient}

Here we consider the flow to be initially at rest and then set in motion by a constant ABG " $\mathrm{K}^{\prime \prime}$. Hence, $\Psi(\tau)$; Eq.(14), subject to BCs. (15) reduces to

$$
\frac{L}{\Delta P} \frac{\partial P}{\partial z}=-K
$$

Therefore, we need to solve the equation 


$$
H \frac{\partial^{2} \Phi}{\partial \tau^{2}}+\frac{\partial \Phi}{\partial \tau}-\left[1+\lambda H \frac{\partial}{\partial \tau}\right]\left[\frac{1}{\eta} \frac{\partial \Phi}{\partial \eta}+\frac{\partial^{2} \Phi}{\partial \eta^{2}}\right]=K,
$$

subject to the boundary and initial conditions

$\phi(1, \tau)=0$, for $\tau \geq 0$,

$$
\phi(\eta, 0)=0, \text { for } 0 \leq \eta \leq 1
$$

Equation (46) can be transformed to a homogenous equation by the assumption

$$
\Phi(\eta, \tau)=\frac{K}{4}\left(1-\eta^{2}\right)-\psi(\eta, \tau)
$$

where $\Psi(\eta, \tau)$ represents the deviation from the steady state solution. Hence,

$$
\left[\frac{\partial}{\partial \tau}\left(1+H \frac{\partial}{\partial \tau}\right)-\left(1+\lambda H \frac{\partial}{\partial \tau}\right)\left(\frac{\partial^{2}}{\partial \eta^{2}}+\frac{1}{\eta} \frac{\partial}{\partial \eta}\right)\right] \psi=0
$$

subject to the boundary and initial conditions

$$
\begin{gathered}
\Psi(1, \tau)=0 \text { for } \tau \geq 0 . \\
\psi(\eta, 0)=\frac{K}{4}\left(1-\eta^{2}\right) \text { for } 0 \leq \eta \leq 1 .
\end{gathered}
$$

Assuming that $\psi(\eta, \tau)=F(\eta) \cdot G(\tau)$, Eq.(49) separates to

$$
\begin{aligned}
& H G^{\prime \prime}+\left(1+\lambda H \alpha^{2}\right) G^{\prime}+\alpha^{2} G=0, \\
& F^{\prime \prime}+\eta^{-1}\left(1+\lambda H \alpha^{2}\right) F^{\prime}+\alpha^{2} F=0 .
\end{aligned}
$$

Equation (52) has the solution, 


$$
G(\tau)=A e^{\gamma} 1^{\tau}+B e^{\gamma} 2^{\tau}
$$

where $\gamma_{1}$ and $\gamma_{2}$ are the roots of the Eq.( 52). On the other hand, Eq. (53) has the solution

$$
F(\eta)=J_{0}\left(\alpha_{m} \eta\right)
$$

Therefore,

$$
\gamma_{1,2}=\frac{-\left(1+\lambda H \alpha_{m}^{2}\right) \pm \sqrt{\left(1+\lambda H \alpha_{m}^{2}\right)^{2}-4 \alpha_{m}^{2} H}}{2 H}
$$

The BCs. $(50,51)$ implies that the constant $\alpha_{\mathrm{m}}$ takes all zeros of the Bessel-function $\mathrm{J}_{0}\left(\alpha_{1}, \alpha_{2}\right.$ .........). Hence,

$$
\begin{gathered}
\Psi(\eta, \tau)=\sum_{m=1}^{\infty} J_{0}\left(\alpha_{m} \eta\right) G(\tau), \\
\therefore \Psi(\eta, \tau)=\sum_{m=1}^{\infty} J_{0}\left(\alpha_{m} \eta\right)\left(A_{m} e^{\gamma_{1 m} \tau}+B_{m} e^{\gamma_{2 m} \tau}\right) .
\end{gathered}
$$

The initial condition (50) and BCs. (51) will not be sufficient to evaluate the constants $A_{m}$ and $B_{m}$. Hence, it is required to employ another condition. We assume that $G(\tau)$ is smooth about the value $\tau=0$ and can be expanded in a power series about $\tau=0$. Assuming $G(\tau)$ to be linear function of $\tau$ in the domain about $\tau=0$, then $G^{\prime \prime}=0$ in Eq. (52). Hence

$$
\begin{gathered}
\left(1+\lambda H \alpha_{m}^{2}\right) G_{m}^{\prime}(0)+\alpha_{m}^{2} G_{m}(0)=0, \\
G_{m}(\tau)=A_{m} e^{\gamma_{1 m} \tau}+B_{m} e^{\gamma_{2 m} \tau},
\end{gathered}
$$

From which we obtain

$$
A_{m}\left[\left(1+\lambda H \alpha_{m}^{2}\right) \gamma_{1 m}+\alpha_{m}^{2}\right]+B_{m}\left[\left(1+\lambda H \alpha_{m}^{2}\right) \gamma_{2 m}+\alpha_{m}^{2}\right]=0
$$


To determine the constants $A_{m}$ and $B_{m}$ we firstly satisfy the remaining condition (51). Owing to Eq. (58) and the initial condition, Eq. (51), we notice that,

$$
\Psi(\eta, 0)=\sum_{m=1}^{\infty}\left(A_{m}+B_{m}\right) J_{0}\left(\alpha_{m} \eta\right)=\frac{K}{4}\left(1-\eta^{2}\right) .
$$

Via the Fourier-Bessel series, Eq. (62) leads to,

$$
A_{m}+B_{m}=\frac{K}{2 J_{1}^{2}\left(\alpha_{m}\right)} \int_{0}^{1} \eta\left(1-\eta^{2}\right) J_{0}\left(\alpha_{m} \eta\right) d \eta
$$

Performing this integration we get

$$
A_{m}+B_{m}=\frac{2 K}{\alpha_{m}^{3} J_{1}\left(\alpha_{m}\right)}-\frac{K}{\alpha_{m}^{2}} \frac{J_{0}\left(\alpha_{m}\right)}{J_{1}^{2}\left(\alpha_{m}\right)} .
$$

From Eqs. (61) and (64) we obtain :

$$
\begin{aligned}
& A_{m}=\frac{\left[\left(1+\lambda H \alpha_{m}^{2}\right) \gamma_{2 m}+\alpha_{m}^{2}\right]}{\left(1+\lambda H \alpha_{m}^{2}\right)\left(\gamma_{2 m}-\gamma_{1 m}\right)}\left[\frac{2 K}{\alpha_{m}^{3} J_{1}\left(\alpha_{m}\right)}-\frac{K}{\alpha_{m}^{2}} \frac{J_{0}\left(\alpha_{m}\right)}{J_{1}^{2}\left(\alpha_{m}\right)}\right], \\
& B_{m}=\frac{\left[\left(1+\lambda H \alpha_{m}^{2}\right) \gamma_{1 m}+\alpha_{m}^{2}\right]}{\left(1+\lambda H \alpha_{m}^{2}\right)\left(\gamma_{1 m}-\gamma_{2 m}\right)}\left[\frac{2 K}{\alpha_{m}^{3} J_{1}\left(\alpha_{m}\right)}-\frac{K}{\alpha_{m}^{2}} \frac{J_{0}\left(\alpha_{m}\right)}{J_{1}^{2}\left(\alpha_{m}\right)}\right] .
\end{aligned}
$$

Finally, the velocity field has the series representation

$$
\begin{gathered}
\phi(\eta, \tau)=\frac{K}{4}\left(1-\eta^{2}\right)-\sum_{m=1}^{\infty} \frac{J_{0}\left(\alpha_{m} \eta\right)}{\left(1+\lambda H \alpha_{m}^{2}\right)\left(\gamma_{2 m}-\gamma_{1 m}\right)}\left\{\left[\left(1+\lambda H \alpha_{m}^{2}\right) \gamma_{2 m}+\alpha_{m}^{2}\right] e^{\gamma_{1 m} \tau}\right. \\
\left.-\left[\left(1+\lambda H \alpha_{m}^{2}\right) \gamma_{1 m}+\alpha_{m}^{2}\right] e^{\gamma_{2 m} \tau}\right\}\left[\frac{2 K}{\alpha_{m}^{3} J_{1}\left(\alpha_{m}\right)}-\frac{K}{\alpha_{m}^{2}} \frac{J_{0}\left(\alpha_{m}\right)}{J_{1}^{2}\left(\alpha_{m}\right)}\right] .
\end{gathered}
$$

The constant-APG velocity field $\varphi(\eta, \tau)$ as a function of $\eta$ shown in Fig. (5). 


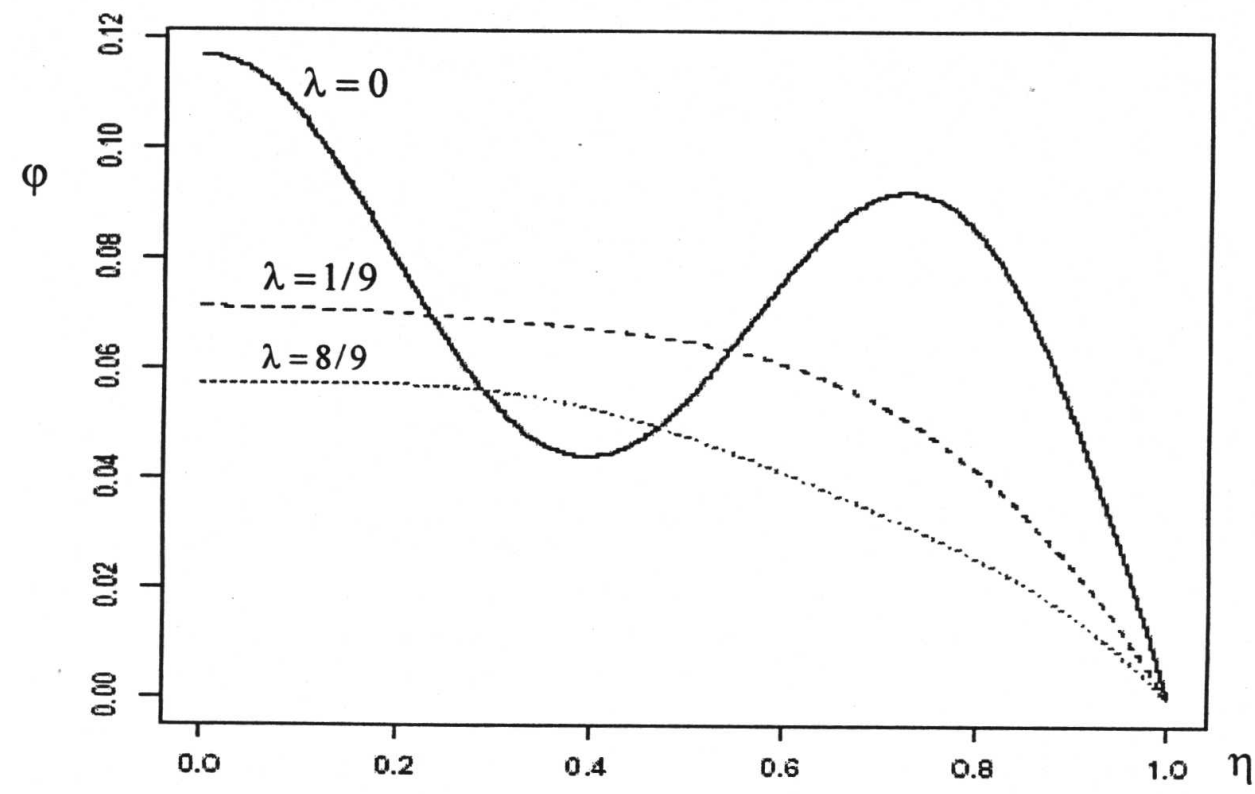

Figure 5. The velocity distribution for constant - APG taking $H=0.2, \tau=0.1$ where the summation is taken for $a_{1}=2.4$, $a_{2}=5.8, a_{3}=8.4$

\section{Results and discussion}

The behavior of $|\beta|$ as a function of $\lambda$ where $H$ is taken as a parameter is shown in Fig. (1). The behavior of $\beta$ is inversely proportional to $\lambda$ while it is fast-decreasing for higher $\mathrm{H}$-values. For any $\beta$-value, the Oldroyd-B fluid exhibits the same form as the UCM-fluid. A close inspection of $\beta^{2}=\alpha^{2}\left(1+\alpha^{2} \mathrm{H}\right) /\left(1+\lambda \alpha^{2} \mathrm{H}\right)$ shows that UCM-fluid is obtained by $\lim _{\lambda \rightarrow 0} \beta^{2}=\beta^{2}$ while $\lim _{\lambda \rightarrow 0} \beta^{2}=\alpha^{2}$ leads to the case of Newtonian fluid. For small values of $|\beta|$ as well as $\mid$ $\beta \eta \mathrm{l}$ and by using the asymptotic expansion of $\mathrm{I}_{0}(\mathrm{x})$, it can be shown that the velocity profiles approaches the parabolic distribution.

For decay-APGs, Figs. (2a) and (2b) show that the velocity profiles of Oldroyd-B and UCM fluids are parabolic for small values of $|\beta \eta|$ while for large $|\beta \eta|$ they are completely different from this situation. The solutions depend on $\eta$ only in the neighboring of the wall. Therefore, such fluids exhibit boundary layer effects [17]].

For pulsating-APG, the velocity distribution is represented in Figs. ( $3 a$ ) and ( $3 b$ ). The smallest value of $\beta$ in both curves is almost parabolic as shown by Eq. (36) while the largest 
value exhibits boundary effect as reviled by Eq.( 43 ). To emphasize the oscillating nature of the solution a three-dimensional diagrams (4a) and (4b) for the smallest and largest values of $|\beta|$ are respectively sketched.

Grigioni, et al [1], studided the behavior of blood as a viscoelastic fluid using the Oldroyd-B model. The results obtained for the velocity distribution stands in agreement with the obtained results in the present work.

\section{Author details}

A. Abu-El Hassan and E. M. El-Maghawry*

*Address all correspondence to: abd_galil@hotmail.com

Physics Department, Faculty of Science, Benha University, Egypt

\section{References}

[1] Grigioni, M., Daniele, C., and D' Avenio, G. The Role of Wall Shear Stress in Unsteady Vascular Dynamics, Vol. 7, No.3 Sep. (2002).

[2] Thurston, G. B., The viscoelasticity of human blood, Biophysical Journal, 12, 1205-1217(1972).

[3] Perspectives in fluid dynamics, A collective introduction to current research, Edited by: Batchelor, G.K., Moffatt, H.K. and Worster, M.G., Cambridge Univ. Press,(2000).

[4] Tucker, C. L., Fundamentals of computer modeling for polymer processing, III, HANSER Publishers, Munich (1989).

[5] Bird, R.B., Curtiss.C.F., Armstrong R.C. and Hassager, O., Dynamics of polymeric liquids, Vol.2, Wiley, New York (1987).

[6] Rahaman, K. D. and Ramkissoon, H., Unsteady axial viscoelastic pipe flows, J.NonNewtonian Fluid Mech., 57 (1995) 27.

[7] Rajagopal, K.R., Int. J. Non-linear Mech. 17 (1982) 369.

[8] Atalik, K. and R. Keunings,R., J. Non-Newtonian Fluid mech. 102, (2002) 299.

[9] Yesilata, B, Fluid Dyn. Res. 31, 41 (2002).

[10] Pontrelli, G., Pulsatile blood flow in a pipe. Computers \& Fluids, 27 (1998) 367.

[11] Pontrelli, G, Blood flow through a circular pipe with an impulsive pressure gradient. Math Models Methods in Appi Sci, 10 (2000) 187 
[12] Bames, H.A., Townsend, P. and Walters, K., Rheol. Acta.10 (1971) 517.

[13] Bames, H.A., Townsend, P. and Walters, K., 244, Nature (1969) 585.

[14] Davies, J.M., Bhumiratana, S. and Bird, R.B. J. Non-Newtonian Fluid Mech, 3 (1977/1978) 237.

[15] Phan-Thien, N. and DudekJ, J.Non-Newtonian Fluid Mech., 11 (1982) 147.

[16] Hayat, T, Asghar, S. and Siddiqui, A.M., Some unsteady unidirectional flows of a Non -Newtonian fluid, Int. J. Eng. Science, 38 (2000) 337.

[17] Schlichting, H., Boundary- layer theory, McGraw-Hill, New York, 1968 